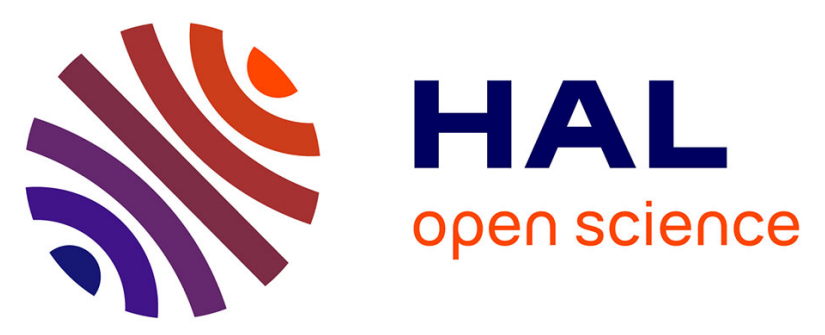

\title{
De l'état d'exception de l'urgence collective au rationnement invisible des politiques de santé publique. Enjeux d'un " continuum du triage"
}

Frédérique Leichter-Flack

\section{- To cite this version:}

Frédérique Leichter-Flack. De l'état d'exception de l'urgence collective au rationnement invisible des politiques de santé publique. Enjeux d'un " continuum du triage ". Les Cahiers du Centre Georges Canguilhem, 2014, 6, pp.67-77. 10.3917/ccgc.006.0067 . hal-01984757

\section{HAL Id: hal-01984757 \\ https://hal.parisnanterre.fr/hal-01984757}

Submitted on 17 Jan 2019

HAL is a multi-disciplinary open access archive for the deposit and dissemination of scientific research documents, whether they are published or not. The documents may come from teaching and research institutions in France or abroad, or from public or private research centers.
L'archive ouverte pluridisciplinaire HAL, est destinée au dépôt et à la diffusion de documents scientifiques de niveau recherche, publiés ou non, émanant des établissements d'enseignement et de recherche français ou étrangers, des laboratoires publics ou privés. 


\author{
DE L'ÉTAT D'EXGEPTION \\ DE L'URGENGE COLLEGTIVE \\ AU RATIONNEMENT INVISIBLE \\ DES POLITIQUES DE SANTÉ PUBLIQUE. \\ ENJEUX D’UN «CONTINUUM DU TRIAGE »
}

Frédérique Leichter-Flack

Depuis quelques années, le terme technique de triage, issu de la médecine d'urgence, est largement sorti de son domaine réservé. Brandi comme une menace, ou convoqué comme un opérateur de lisibilité destiné à éclairer de sa lumière crue des choix de priorisation budgétaire opaques, il se laisse repérer, explicite ou implicite, dans un grand nombre de débats politiques sur l'allocation des ressources en santé publique, dans l'humanitaire ou dans l'action sociale, y compris donc en dehors du seul champ de l'urgence médicale. Cet usage « extensif » de la référence au triage s'observe surtout aux États-Unis d'Amérique : dans les médias américains, les pratiques de triage, de sélection et de rationnement des ressources, se fraient un chemin comme des sujets d'intérêt public. Mais qu'implique ce partage d'enjeux généralement réservés aux spécialistes? Quelles sont, dans les différents contextes concernés, les implications de cette appréhension large du triage? Quelle pertinence ont ces références parfois très éloignées du strict contexte de l'urgence médicale et de ses enjeux de vie ou de mort imminents? Et plus profondément, est-il utile et fécond, sur le plan éthique, de penser le triage en continuum? Jusqu'où encore étendre ce continuum, en aval des situations d'urgence 
médicale collective qui ont servi à en formuler les premiers protocoles, mais aussi en amont d'elles, vers des expériences historiques qui ont contraint des groupes humains à s'organiser quand il n'y avait "pas assez de vie » pour tout le monde, ou vers des situations fictionnelles artificiellement fabriquées pour éprouver les réactions des hommes placés en situation extrême? Telles sont les questions que cet article voudrait soulever.

\section{PARCOURIR LES NOUVELLES GAMMES DU TRI}

Partons de quelques exemples de ces usages extensifs du «triage » et du « rationnement », et des efforts de vulgarisation de ces notions. En 2009, une longue enquête parue dans le New Tork Times offre à l'attention publique une affaire de triage qui a mal tourné, dans un hôpital inondé de la Nouvelle-Orléans dévastée par le cyclone Katrina en 2005. Une dizaine de corps retrouvés sur place après l'évacuation complète de l'hôpital avait semé le soupçon : des médecins avaient-ils pris la décision d'euthanasier certains patients dont l'évacuation leur paraissait trop difficile, afin d'éviter de les abandonner vivants sur place ? Un non-lieu avait finalement refermé le volet judiciaire de l'affaire, mais c'est le fossé entre l'expérience des spécialistes de la catastrophe, et les perceptions ordinaires du grand public, que la journaliste Sheri Fink a voulu explorer, en se lançant dans une minutieuse reconstitution des cinq jours vécus à Memorial après le passage du cyclone - de l'intérieur même de ce huis clos qu'était devenu l'hôpi$\mathrm{tal}^{1}$. Si les situations de catastrophe font imploser les protocoles

1. Sheri Fink, Deadly choices at Memorial, NYTimes, 30 août 2009 (http:// www.nytimes.com/2009/08/30/magazine/30doctors.html ?pagewanted=all.

Consulté le 15 octobre 2013). Voir aussi Sheri Fink, Five Days at Memorial. Life and Death in a Storm-Ravaged Hospital, Crown, 2013. Pour une analyse de l'affaire Memorial, voir F. Leichter-Flack, Sauver ou laisser mourir. Catastrophe et éthique médicale, 25 avril 2011, dans la revue La Vie des Idées (http://www.laviedesidees.fr/Sauver-oulaisser-mourir.html. Consulté le 15 oct. 2013). 
d'éthique de l'urgence médicale, le triage d'urgence médicale doit-il être adapté à l'avance aux contextes extrêmes? Comment repenser la responsabilité pénale et morale des médecins décisionnaires ${ }^{1}$ ? L'effort de vulgarisation de l'expérience des médecins de Memorial, auquel la journaliste se risque dans son enquête, attise le débat sur les conditions et les critères devant régir le triage de catastrophe - et donc aussi sur les différences entre le triage d'urgence médicale «banale », et le triage de catastrophe. Les critères presque exclusivement médicaux $\mathrm{du}$ premier ne posent pas problème au grand public, qui trouve logique de faire attendre les blessés qui le peuvent pour soigner en priorité les blessés graves ; mais la catastrophe ne suppose-t-elle pas, dans un réflexe utilitariste, de faire exactement l'inverse, de réserver toutes les ressources pour ceux qui ont une chance de s'en tirer, en faisant attendre, et éventuellement en laissant mourir, ceux dont les chances de survie sont moindres? Ne faudrait-il pas alors mobiliser le grand public pour qu'il donne son avis sur la manière dont il souhaiterait être traité en pareil cas? Par bien des aspects, en effet, les situations d'urgence collective exceptionnelle, avec pénurie de moyens, pourraient bien ne plus relever de la même catégorisation que les pratiques de triage d'urgence médicale « ordinaire ».

Cette question a été reposée ces dernières années à l'occasion des menaces de pandémie grippale. Dans la plupart des pays, les protocoles de priorisation des ressources médicales (soins, médicaments, vaccins) préparés en prévision de pandémies de grande ampleur, sont restés l'apanage d'experts, mais certains États américains ont pris au sérieux l'enjeu de justice démocratique, jusqu'à solliciter l'avis spontané du grand public sur les logiques de priorisation et les hiérarchies de valeurs à mettre en œuvre. Divers exercices de public engagement (visant, par la sollicitation qualitative de panels représentatifs, à

1. Dans le cas de l'hôpital Memorial étudié par Sheri Fink, c'est sur la responsabilité des médecins eu égard à la décision d'euthanasier des patients que la justice a eu à se prononcer, mais la défense a justement cherché à déplacer le débat sur le terrain de la responsabilité des médecins eu égard aux critères et méthodes de triage retenus dans cette situation d'urgence exceptionnelle. 
prendre le pouls du grand public sur une affaire dont il faut qu'il se saisisse puisqu'il est au premier chef concerné) ont ainsi vulgarisé les dilemmes éthiques surgissant de la double nécessité de penser des logiques de priorisation en amont de la catastrophe à prévoir, et de respecter la pression démocratique de l'égalité de valeur des vies.

Or, avec la pandémie (et sa forte part d'aléa, tant sur le plan des risques de contamination que sur celui des chances de guérison), le triage sort en grande partie du seul champ de l'expertise médicale : dans les exercices de public engagement, des expériences de pensée sont conçues pour mesurer les choix spontanés des gens entre des profils de patients socialement bien identifiés (âge, métier, statut familial, etc.) et qui recevraient un bénéfice équivalent (avec le même degré d'incertitude) de l'accès en réanimation ${ }^{1}$. S'il est impossible de dire qui profitera le plus de l'accès aux soins sur le plan médical, il est possible de dire avec quasi-certitude que ceux à qui on n'offre pas leur chance sont condamnés. Le triage devient une sorte de gestion humaine de l'arbitraire du sort, une correction de l'injustice aléatoire de la contamination... Mais selon quels critères opérer? La pandémie articule plusieurs sens du triage, plusieurs questions afférentes à ces logiques de priorisation, de sélection, et somme toute, d'exclusion : qui protéger (une fois le vaccin disponible) et qui laisser exposer ; à qui donner une chance de survie potentielle (par l'accès en réanimation) et à qui la dénier? Les théories de la justice se retrouvent alors confrontées aux limites de leur pertinence sur le terrain, face à la singularité toujours renouvelée des situations.

C'est un écueil similaire qu'interroge un reportage de la radio américaine au moment du séisme de 2010 à Haiti : le dilemme des urgentistes américains dépêchés sur place face au cas d'une jeune femme en insuffisance respiratoire chronique qu'on renoncera à secourir, pour réserver l'oxygène disponible aux nombreuses victimes qui, suite au séisme, en ont besoin chacune un peu et peu de temps, attire l'atten-

1. Voir par exemple, en ligne, le rapport suivant : http://s3.amazonaws.com/ propublica/assets/docs/seattle_public_engagement_project_final_sept2009.pdf. Consulté le 15 oct. 2013. 
tion du grand public sur les sacrifiés du triage opéré par les services de secours américains ${ }^{1}$. L'application d'un critère d'efficacité utilitariste peut conduire à des protocoles impliquant de refuser de soigner - c'est-à-dire de laisser mourir - ceux qui auraient besoin de beaucoup de ressources médicales pour leur seul cas... Ce qui amène à se poser la question essentielle : pourquoi est-on là, sur place, au lendemain du séisme? Pour sauver toutes les vies qui peuvent l'être? Ou pour sauver, avec un stock de ressources limitées, le plus de vies parmi celles (seulement ?) que la catastrophe a menacées (autres pathologies, s'abstenir...) ? Si le triage de l'aide humanitaire d'urgence, après une catastrophe naturelle, vise, par définition, le retour le plus rapide possible à la situation "normale » d'avant la catastrophe, quelle sorte de résignation ontologique à l'injustice des sorts ce « retour à l'ordinaire » recouvre-t-il ?

Un autre contexte de recours à l'imaginaire du triage dans le débat public américain - le plus fréquent - nous est fourni par la récente réforme de la santé. Les détracteurs de la réforme ont usé et abusé de l'imaginaire tragique du triage pour agiter la menace d'un « rationnement » arbitraire et antilibéral de la santé : le modèle britannique de rationnement des soins coûteux et ses raisonnements mobilisant l'unité de mesure QALY ( Quality Adjusted Life Year ») ont ainsi été convoqués pour faire le portrait de victimes du triage, dans le dessein avoué d'invalider la prétention de justice sociale de l'Obamacare. En exploitant l'émotion facilement créée autour de quelques anecdotes, les détracteurs de la réforme de la santé valident bien sûr sans le dire un autre type de tri de la santé, implicite celui-là, fondé sur les logiques de marché. Puiser dans l'imaginaire tragique du triage - brandi ici comme un épouvantail et un repoussoir - est pour eux un moyen de clore le débat plutôt que de l'ouvrir.

$\mathrm{Au}$ contraire, l'importante série radiophonique "Rationing Health » (PRI's the World) visait l'effet inverse : si elle choisit d'ouvrir le champ du triage dans plusieurs directions très différentes, c'est pour

1. Voir http://pri.org/stories/2010-02-23/doctors-face-ethical-decisions-haiti. Consulté le 15 oct. 2013. 
mieux convaincre le public profane de la nécessité démocratique de s'approprier ce débat ${ }^{1}$. La série évoque en effet quatre contextes dans lesquels un rationnement de l'accès aux ressources de santé vitales est opéré : la gestion du pic pandémique en Inde, à travers un reportage sur les dilemmes d'une pédiatre indienne que la pénurie de matériel de soin contraint à choisir auquel des bébés gravement atteints elle accordera l'opportunité du dernier respirateur artificiel disponible dans le service; l'attribution des dialyses en Afrique du Sud, assujettie à la décision de commissions médicales examinant le dossier complet (médical et social) de chaque patient ; l'agence NICE (National Institute for Health and Care Excellence) en Grande-Bretagne, qui, sur la base de raisonnements convertis en unités QALY, accorde ou non la prise en charge de traitements coûteux - avec l'exemple d'un patient cancéreux qui s'est vu refuser le traitement qui aurait pu prolonger son existence de quelques mois; et, en Zambie, la distribution des trithérapies contre le VIH/sida sur une base de "first come first serve ", procédure qui, sous des allures d'accès libre ouvert à tous, régit un rationnement implicite qui discrimine socialement ceux qui ne peuvent pas se permettre de consacrer une journée par mois à faire la queue. Par ces quatre coups de projecteur, la série cherche à éclairer le débat sur les politiques de santé en montrant que le rationnement et le triage sont des réalités repérables dans tous les contextes de santé publique, à des titres différents et selon des critères et méthodes spécifiques à chaque fois, mais comparables, et qu'il vaut mieux, pour des raisons de justice sociale, qu'ils soient alors publiquement discutés, régulièrement réévalués, et démocratiquement validés par une pleine implication en conscience de tous les acteurs concernés. Loin de militer contre tout rationnement, ou de s'effaroucher du concept, les auteurs de la série cherchent à le rendre visible et familier à tous sous toutes ses formes, afin d'améliorer la justice des systèmes en jeu, de

1. La série est une coproduction BBC, PRI et WGBH, avec le soutien d'un financement de la Bill and Melinda Gates Foundation. Les contributeurs sont S. Fink, D. Wickler, P. Cox et D. Baron. Voir http://www.rationinghealth.org (déc. 2010). Consulté le 15 oct. 2013. 
dénoncer leurs effets pervers éventuels, et de documenter leurs effets de seuil et leurs angles morts. C'est la raison sans doute pour laquelle, dans chacun de ces reportages destinés au grand public, c'est surtout par le biais des «sacrifiés » du triage (les non-évacués, les privés de traitement, les derniers sur les listes d'attente, etc.) que ces choix tragiques sont évoqués. L'insistance sur les morituri - ceux que l'on considère comme non prioritaires au point parfois de les abandonner à la mort - rappelle le caractère tragique des arbitrages de santé publique, ou le réveille, même quand il est dissimulé derrière des décisions très en amont de l'imminence de mort.

\section{RÉVEILLER LES FANTÔMES DU TRIAGE. DILEMMES HISTORIQUES ET FICTIONNELS EN SITUATION EXTRÊME}

Des situations de catastrophe et d'urgence collective, aux arbitrages budgétaires plus ordinaires, un continuum du triage semble donc pouvoir être dessiné, le long d'une échelle où la décision de vie ou de mort paraît plus ou moins nette ou floue, imminente ou lointaine, directe ou indirecte. Une même unité de mesure peut circuler des situations les plus extrêmes où les choix engagent directement qui vivra et qui mourra, vers des répartitions de budgets aux conséquences humaines moins immédiatement perceptibles en termes de vie ou de mort. Certes, l'usage que l'on peut en faire ne va pas de soi : assigner des conséquences mortelles à des choix abstraits de priorisation budgétaire peut relever du coup de force argumentatif. Mais ce continuumlà ne poserait pas tant question si ne planait sur lui une ombre, un « fantôme » qui constitue l'amont du triage. Il s'agit de désigner ainsi toutes ces références, dans l'imaginaire collectif - souvenirs et expériences historiques traversées, ou fictions grand public -, où, en situation extrême, la question de « qui sauver quand on ne peut pas sauver tout le monde » s'est posée sans que le recours à une expertise médicale (état clinique, pronostic...) ne soit une option de guidance pos- 
sible. Le paradigme qui s'impose alors à l'esprit est celui des naufrages : quand il n'y a pas assez de places pour tous les passagers dans les canots de sauvetage, qui doit y monter et qui doit laisser sa place? Et que faire quand le canot surchargé risque de couler sous le poids des naufragés en trop?

Mais l'histoire du $\mathrm{XX}^{\mathrm{e}}$ siècle a fourni d'autres exemples reformulant à une échelle massive les dilemmes d'allocation de vie ou de mort. Retracer le continuum du triage dans l'autre sens suppose non plus d'aller de la catastrophe d'urgence collective vers les choix opérés en situation ordinaire, mais de la catastrophe pensée comme une contrainte "naturelle » et subie, vers des situations extrêmes où les dilemmes d'allocation de la rareté vitale sont le fruit de volontés malveillantes et perverses : évacuation ou exfiltration clandestine de quelques-uns au milieu d'une population en danger d'extermination ou de génocide, organisation de réseaux de sauvetage restreints, gestion des contingents de travail forcé ou de déportation par les conseils juifs des ghettos sous l'occupation nazie, négociation autour de la constitution de listes de protégés et, bien sûr, sélections au sens nazi du terme... Dans toutes ces situations, l'imaginaire de la rareté vitale est fabriqué artificiellement comme si c'était le fruit du mal physique, alors même que c'est celui du mal moral...

Davantage : si l'on réveille les fantômes du triage, d'autres images en surimposition peuvent s'inviter dans le débat. Car la fiction a pris le relais, dans une large mesure, de cet imaginaire de la pénurie de vie produit par la terreur concentrationnaire nazie que la littérature de témoignage et l'historiographie nous ont transmis. La fiction donne à imaginer et à sentir le triage en inventant des vignettes de «réalité augmentée » : ainsi du célèbre "choix de Sophie » qu'un médecin nazi, dans le roman de William Styron, impose à une mère, entre ses deux enfants, sur le quai d'Auschwitz ${ }^{1}$. Mais la fiction ne se limite pas au contexte de l'appareil de terreur nazie : elle travaille aussi d'autres contextes, soit réalistes, soit fantastiques ou de science-fiction, où les dilemmes de rareté vitale sont déplacés et servent de support à une

1. William Styron, Sophie's Choice, New York, Random House, 1979. 
réflexion globale sur les valeurs et les lignes rouges de notre société. Dans l'un et l'autre cas, la fiction tire son attrait de la fascination produite par ces expériences d'horreur morale qu'elle nous propose de vivre par procuration ${ }^{1}$.

Faut-il donc voir et faire voir partout du tri ? L'usage « en continuum » du concept est-il rendu inéluctable par les choix de vocabulaire, qui emportent des associations d'idées réflexes et déclenchent des analogies spontanées dans les perceptions du grand public, réveillant alors des traumatismes historiques ( sélection », "rationnement ») ? Ou bien correspond-il à un objectif d'engagement public et de mobilisation citoyenne destiné - par l'insistance sur la part non médicale des enjeux de triage - à élargir l'assise des décideurs, du champ de l'expertise médicale ou éthique, à celui du grand public? Les analogies opérées par l'usage en continuum du concept de triage sont-elles utiles ou nocives? Constituent-elles une occasion d'éveil au débat public et un instrument au service de l'exigence d'une plus grande justice sociale, ou le passage à la «puissance tragique » (à la « puissance triage »?) a-t-il des effets pervers?

\section{ENJEUX, RISQUES ET INTÉRÊTS \\ DU « PASSAGE À LA PUISSANGE TRIAGE »}

Il est certain que l'insistance sur l'angle mort du tragique concourt à obliger l'ensemble des citoyens concernés à se réapproprier les décisions en les sortant du domaine réservé de l'expertise. Le passage à la «puissance triage » alerte sur les limites et les risques de certains choix publics insuffisamment discutés; son pouvoir de clarification des enjeux en termes d'éthique et de justice sociale est incontestable. Ainsi de la formulation au prisme du triage des questions soulevées par les politiques publiques d'incitation au dépistage anténatal, ou du débat

1. Je renvoie aussi sur ce point à la conclusion de mon Laboratoire des cas de conscience, Paris, Alma, 2012. 
sur l'allocation des fonds collectés à l'occasion du Téléthon (inventer des thérapies et améliorer la qualité de vie des personnes atteintes? $\mathrm{Ou}$ mieux dépister pour éviter la naissance de futurs malades?). Cependant, lancer le débat sous l'angle du triage et du rationnement, alors que règne une approche coutumière bien acceptée par le grand public, comporte des risques. Réinjecter du tragique dans la vie ordinaire c'est risquer de rompre les équilibres existants sans certitude qu'un nouveau consensus à moindre coût social pourra être trouvé. Il n'est du reste pas garanti que l'accent soit alors mis sur la nécessité de débattre publiquement des critères acceptables. La médiatisation des «sacrifiés du triage » peut ainsi conduire, en rendant intolérable le tragique de ces arbitrages de vie ou de mort, à des réflexes d'évitement pas toujours pertinents pour le bien-être collectif, comme par exemple la décision de supprimer la rareté en augmentant, au détriment d'autres causes, les ressources disponibles en amont de leur répartition. Inversement, la fascination pour le tragique des décisions de vie ou de mort peut pousser, au contraire, à faire passer pour une condition existentielle naturelle la quantité de ressources à distribuer, alors même que ce curseur du tragique est entre nos mains.

La réflexion publique sur les critères est rendue d'autant plus difficile qu'aujourd'hui la frontière se brouille entre les catégories de discrimination jugées acceptables (l'état de santé, le pronostic) et celles jugées injustes (la richesse, le statut social et la capacité d'influence, la race). Ces catégories se mêlent non seulement à cause des corrélations évidentes, impossibles à ignorer, entre les deux familles de critères (un riche qui pourra se payer du repos et des soins supplémentaires aura un pronostic meilleur qu'un pauvre dont les conditions de vie et de travail sont moins favorables à la récupération), et à cause de l'influence des conditions sociales et économiques sur la répartition des maladies et sur les chances de chacun face à la vie et la mort, mais aussi, plus profondément, en raison d'une poussée de l'exigence de justice sociale sur le terrain anciennement dévolu à la nature. L'état de santé, utilisé comme critère médical pour asseoir un pronostic ne serait ainsi que l'autre nom de notre résignation à l'impuissance médicale : si l'on tient compte de tout ce qu'il est possible de tenter pour 
repousser toujours plus loin les limites de la condamnation à mort du destin qu'est pour nous la maladie, avec les progrès des technologies médicales (disponibles contre frais, ou envisageables sous forme d'essais cliniques innovants comportant risques et chances), le critère du pronostic a-t-il encore une légitimité dans les pratiques de triage? Dans quelle mesure les critères médicaux ne sont-ils pas la couverture de notre résignation à l'inégalité des chances?

La résignation face aux contraintes objectives du sort, de la nature ou de la providence, qui a prévalu dans l'invention du modèle du triage militaire sur le champ de bataille, ne tient plus dans un monde où tout est choix et décision humaine, et où la providence n'est plus acceptée comme un modèle légitime de répartition des sorts. Retracer alors tout cet amont du triage, ce réservoir de références, souvenirs, situations et d'expériences éventuellement fictionnelles qui animent l'imaginaire collectif, doit aussi permettre de réinterroger la part de l'expertise médicale dans les logiques éthiques de triage. Car ces variations, historiques et fictionnelles, font intervenir des choix de priorisation des vies dans lesquelles le pronostic, l'autre nom de notre impuissance face aux forces de la nature et du destin, n'est plus d'aucun recours. Face au mal (incarné par la pénurie de vie), l’imaginaire du triage non médical nous renvoie à nos responsabilités : non plus comment combattre le mal, mais comment le gérer, comment le répartir, comment en décider... Penser le triage en continuum enlève ainsi le tragique à l'antique confrontation entre le destin (sort ou providence) et la volonté humaine, pour le situer tout entier sur le terrain de la confrontation des volontés humaines. 